\title{
Anschriften der Autorinnen
}

\author{
Ahrendt, Cordula \\ Lehrhebamme \\ Berufsfachschule an der Medizinischen \\ Akademie \\ Erich-Weinert-Str. 3 \\ D-39112 Magdeburg
}

Alef, Ursula

Lehrhebamme/Schulleitung

Hebammenschule

Kliniken St. Antonius GmbH

Vogelsangstr. 106

D-42106 Wuppertal

Bludau, Christine

freiberufl. Hebamme

Allerweg 88

D-30851 Langenhagen

Eichenberger, Dorothée

Hebamme/Berufsschullehrerin

f. Hebammen

Hebammenschule Universitätsspital

Huttenstr. 46

CH-8091 Zürich

Frank, Elisabeth

Lehrhebamme/Schulleitung

Hebammenschule der

Georg-August-Universität

Humboldtallee 11

D-37073 Göttingen

Friedrich, Jule

freiberufl. Hebamme/

Diplom-Gesundheitsmanagerin

Fichtenkamp 26

D-22393 Hamburg

Frühauf, Johanna

freiberufl. Hebamme/Politologin/

Pro Familia-Mitarbeiterin

Friedrichsplatz 9

D-35037 Marburg
Geist, Christine

Lehrhebamme

Hebammenschule

Krankenhaus Neukölln

Mariendorfer Weg 28

D-12051 Berlin

Gorontzy, Dagmar

Lehrhebamme

Hebammenschule Krankenhaus Neukölln

Mariendorfer Weg 28

D-12051 Berlin

Halbach, Martha

Lehrhebamme/Schulleitung

Hebammenschule Luisenhospital

Boxgraben 99

D-52064 Aachen

Harder, Ulrike

Lehrhebamme/Schulleitung

Hebammenschule Martin-Luther-Krankenhaus

Caspar-Theyß-Str. 27-33

D-14193 Berlin

Hauser, Regula

Hebamme/Ausbilderin

Hebammenschule Universitätsspital

Huttenstr. 46

CH-8091 Zürich

Heckelen, Ulrike

freiberufl. Hebamme

Alteburger Str. 32

D-50968 Köln

Herber, Astrid

Lehrhebamme/Schulleitung

Hebammenschule der

Städt. Kliniken Kassel

Mönchebergstr. 41/43

D-34125 Kassel

Hezel, Dorothea

freiberufl. Hebamme/Stud. Päd.

Alteburger Str. 32

D-50968 Köln 
Hübel, Andrea

freiberufl. Hebamme

Hebammenpraxis Rostock

Goethestr. 20

D-18055 Rostock

\section{Kahf, Sigrun}

Lehrhebamme

Hebammenschule

Krankenhaus Neukölln

Mariendorfer Weg 28

D-12051 Berlin

Kerkmann, Marianne

Leitende Kreißsaalhebamme/

Schulleitung

Staatl. Berufsfachschule für Hebammen an der 1. Universitäts-Frauenklinik

Maistr. 11

D-80337 München

Kimmerle, Edith

Lehrhebamme/freiberufl. Hebamme

Neugasse 2

D-64372 Ober-Ramstadt

Kirchner, Simone

Lehrhebamme/freiberufl. Hebamme

Urbanstr. 30

D-10967 Berlin

Kluge, Susanne

freiberufl. Hebamme/Diplom-

Sozialpädagogin

Mörikestr. 16

D-34125 Kassel

Krauss, Sabine

freiberufl. Hebamme

Böhmestr. 19

D-27283 Verden/Aller

Kriegerowski-Schröteler, Gisela

Lehrhebamme/Schulleitung

Hebammenschule

Krankenhaus Neukölln

Mariendorfer Weg 28

D-12051 Berlin

Lippens, Frauke

freiberufl. Hebamme

Krockmannstr. 5

D-22299 Hamburg
Lübke, Marion, Dr. med.

Hebamme/Ärztin

Universitäts-Klinikum Rudolf Virchow

Standort Charlottenburg

Pulsstr. 4

D-14059 Berlin

Luyben, Ans

Hebamme/Berufsschullehrerin für

Gesundheitsberufe

Universitätsspital Bern

Schanzeneckstr. 1

CH-3012 Bern

Mailänder, Ina

angestellte/freiberufl. Hebamme

Vinzenz Pallotti Hospital

D-51406 Bergisch Gladbach/Bensberg

Orlowski, Margarete

Lehrhebamme/freiberufl. Hebamme

Schule für Medizinalfachberufe

Charité, Fachbereich Geburtshilfe

Schumannstr. 18

D-10117 Berlin

Polleit, Heike

freiberufl. Hebamme/Pro Familia-

Mitarbeiterin

Markgrafenstr. 51

D-78467 Konstanz

Presting, Ulrike

Hebamme/Juristin

Schützenstr. 15

D-12165 Berlin

Rettenegger-Wagner, Susanne

freiberufl. Hebamme

Thurnfelsstr. 37

A-6176 Völs

Rockel-Loenhoff, Anna

Hebamme/Ärztin/Dozentin

Birkenweg 11

D-59425 Unna

Rodriguez, Josepha

Hebamme/Ärztin

Universitäts-Klinikum Rudolf Virchow

Standort Charlottenburg

Pulsstr. 4

D-14059 Berlin 
Rosenberger, Christl

freiberufl. Hebamme/Berufsschullehrerin für Gesundheitsberufe SRK

Hebammenschule St. Gallen

Postfach 188

CH-St. Gallen

Roth, Susanna

freiberufl. Hebamme

Nördliche Auffahrtsallee 28

D-80638 München

Schilling, Rosa Maria

Hebamme/ Diplom Medizinpädagogin

Schule für Medizinalberufe

Charité, Fachbereich Geburtshilfe

D-14167 Berlin

Schippers, Gabriele

freiberufl. Hebamme/Diplompsychologin

Freiheitstr. 11

41352 Korschenbroich

Schirren, Cornelia

Lehrhebamme

Pfalzburger Str. 52

D-10717 Berlin

Schoppa-Remm, Antje

freiberufl. Hebamme

Mainzer Str. 38

D-56068 Koblenz

Schwarz, Clarissa

freiberufl. Hebamme/Lehrerin/

Stud. Public Health

Markgrafenstr. 5

D-10969 Berlin

Steffen, Gisèle

freiberufl. Hebamme

Wiesenstr. 36

D-35641 Schöffengrund 2
Steininger, Ilse

freiberufl. Hebamme/Lehrerin für Geburtshilfe

Hebammenschule Kanton Frauenspital

Schanzeneckstr. 1

CH-3012 Bern

Stiefel, Andrea

Lehrhebamme/Hebammenschule

Martin-Luther-Krankenhaus

Caspar-Theyß-Str. 27-33

D-14193 Berlin

Szász, Norá

Hebamme/Ärztin

Krankenhaus Am Urban

Dieffenbachstr. 1

D-10967 Berlin

Tegethoff, Dorothea

freiberufl. Hebamme

Britzer Str. 58

D-12109 Berlin

Teich, Mareike

Hebamme/OP-Schwester

Hebammenschule am Städt. Klinikum

Celler Str. 38

D-38114 Braunschweig

Warbanov, Renate

Leitende Kreißsaalhebamme

Universitätsklinikum Charité

Schumannstr. 18

D-10117 Berlin

Wepler, Doris

freiberufl. Hebamme/Geburtshaus Berlin

Garde-du-Corps-Str. 4

D-14059 Berlin

Wöste, Stephanie

Hebamme/Diplompsychologin

Baron Voght Str. 96

D-22609 Hamburg 
